\title{
Collective effect of hydrogen in argon and Mg as ambiance for the heat treatment on $\mathrm{MgB}_{2}$
}

\author{
B. B. Sinha ${ }^{a}$, S. H. Jang ${ }^{b}$, and K. C. Chung*, \\ ${ }^{a}$ National Center for Nanoscience and Nanotechnology, Mumbai University, Santacruz(E), Mumbai 400098 \\ ${ }^{\mathrm{b}}$ Korea Institute of Materials Science, Changwon, Korea
}

(Received 9 June 2014; revised or reviewed 19 June 2014; accepted 20 June 2014)

\begin{abstract}
Magnesium diboride superconductor is still of considerable interest because of its appealing characteristics towards application mainly at around $20 \mathrm{~K}$. Unlike Nb-based superconductors, $\mathrm{MgB}_{2}$ can be operated by cryogen-free cooler which provides a cost effective alternative at low field of around 2-5 T. To explore this operating field region considerable efforts are necessary to marginally improve the superconducting properties of $\mathrm{MgB}_{2}$. Under this situation, even the heat treatment environment during the synthesis is considered as an important factor. The addition of $\mathrm{H}_{2}$ gas in small amount with $\mathrm{Ar}$ as a mixed gas during annealing has an adverse effect on the superconducting properties of $\mathrm{MgB}_{2}$. It is although interesting to find that the presence of Mg vapor along with hydrogen during heat treatment results in the appreciable improvement in the flux pinning and the overall response of the critical current density for the ex-situ $\mathrm{MgB}_{2}$ samples.
\end{abstract}

Keywords: Electric resistance, Magnetic fields, $\mathrm{MgB}_{2}$ superconducting material, Critical current density

\section{INTRODUCTION}

Magnesium diboride superconductor has been observed to be highly sensitive to sample preparation history and composition. Since the discovery of superconductivity in this compound [1], $\mathrm{MgB}_{2}$ has been studied by various synthesis techniques through different processes with the different levels of superconducting properties [2-6]. $\mathrm{MgB}_{2}$, being a binary intermetallic compound, was anticipated to be an easily synthesizable compound. But the vast difference in melting point of starting materials, $\mathrm{B}$ and $\mathrm{Mg}$ make this synthesis process somewhat difficult. It showed widely different properties depending upon the synthesis process and the precursor used. A convenient example was presented in ref.[7] wherein the resistivity of various samples prepared by the different synthesis techniques is compared. It was surprising to see that the samples show $39 \mathrm{~K}$ of superconducting transition $\left(T_{\mathrm{c}}\right)$ even with the room temperature resistivities of little over $100 \mathrm{~m} \Omega-\mathrm{cm}$ [8] and also with less than $10 \mu \Omega$-cm [9]. Different level of grain connectivity among the superconducting grains depending upon the extent of impurities, secondary phases, and stoichiometry is anticipated as the reason for the discrepancies in the superconducting parameters. Various research work in this field had made it quite clear that unlike high $T_{\mathrm{c}}$ cuprate superconductors there is a strong inter-granular current flow in $\mathrm{MgB}_{2}$ superconductor [10-12]. Keeping away the defects and grain boundary effects, the improvement of the critical current density in pure $\mathrm{MgB}_{2}$ is highly related with the enhancement in the phase formation and the sample density [13]. Thus, the main parameter behind the discrepancies in various $\mathrm{MgB}_{2}$

* Corresponding author: kcchung@kims.re.kr samples is the high vapor pressure of $\mathrm{Mg}$ which results in the instability and further depletion of Mg content. In this work, the research to improve the superconducting property of $\mathrm{MgB}_{2}$ merely by using a combination of hydrogen and $\mathrm{Mg}$ annealing ambiance is carried out. $\mathrm{MgB}_{2}$ powder was used as a precursor towards nullifying the effect of preparative conditions in the synthesis of in-situ $\mathrm{MgB}_{2}$.

\section{EXPERIMENTAL}

To study the collective effect of $\mathrm{H}_{2}$ and $\mathrm{Mg}$ ambiance on ex-situ $\mathrm{MgB}_{2}$ samples, a commercially available magnesium diboride powder with the particle size of 100 Mesh was used. The precursor powder was hand-milled for about two hours in the agate mortar under the environment of ultra high pure $\mathrm{Ar}$ in glove box. This hand-milled powder was then transferred in to mould of $10 \mathrm{~mm} \times 10$ $\mathrm{mm}$ to form a pellet under the applied pressure of 10 $\mathrm{Ton} / \mathrm{cm}^{2}$. This pellet so formed was placed in Fe tube for the annealing in a tube furnace at $900{ }^{\circ} \mathrm{C}$ for 3 hours. The temperature was ramped up at a rate of $15{ }^{\circ} \mathrm{C} / \mathrm{min}$ and different gas was flowed depending upon samples at flow rate of $2 \mathrm{~L} / \mathrm{min}$. The ambiance used during annealing was $\mathrm{Ar}, \mathrm{Ar}+4 \% \mathrm{H}_{2}, \mathrm{Ar}+\mathrm{Mg}$, and $\mathrm{Ar}+4 \% \mathrm{H}_{2}+\mathrm{Mg}$ and the samples were accordingly named as $\mathrm{Ar}, \mathrm{ArH}, \mathrm{ArMg}$, and ArHMg, respectively. $\mathrm{Ar}+4 \% \mathrm{H}_{2}$ is a mixed gas of ultra high pure and used as purchased. It was also designed to maintain an equilibrium ambiance of $\mathrm{Mg}$ around samples in a way that there is no built-up of Mg pressure in Fe tube. Hence the Fe tube was provided with a small hole at one side. This would create an equilibrium pressure with the 
environment outside the Fe tube that would prevent the formation of excess $\mathrm{Mg}$ pressure (2.93 Torr at $650{ }^{\circ} \mathrm{C}$ [14]) and the forceful infiltration of $\mathrm{Mg}$ into the samples. Amount of $\mathrm{Mg}$ powder introduced was equal to the weight of the pellet (which in this case $157 \mathrm{mg}$ ) and was placed at a sufficient distance from the pellet. The pellet samples were characterized by X-ray diffraction (XRD) technique to study the structural variations. It was performed using Rigaku, D/Max 2200 system with $\mathrm{Cu} \mathrm{K} \alpha$ radiation. Also, Field Emission Scanning electron microscopy (FE-SEM) (using HITACHI FE-SEM S-4700) was carried out to study the effect of annealing ambiance on the microstructure of samples. The superconducting transition temperatures of samples were measured through the temperature dependent resistivity using a four probe method. The zero field cooled (ZFC) and field cooled (FC) measurements was also done to confirm and compare the superconducting properties of the samples. The measurement of the magnetization as a function of magnetic field was obtained to calculate the critical current density using the modified Bean's formula [15] as given below:

$$
J_{c}=\frac{20 \Delta M}{a\left(1-\frac{a}{3 b}\right)}
$$

Where, $J_{c}$ is critical current density in $\mathrm{A} / \mathrm{cm}^{2}, \Delta \mathrm{M}$ is the difference in the magnetization in emu $/ \mathrm{cm}^{3}$ for increasing and decreasing field, ' $a$ ' and ' $b$ ' is the thickness and width of the sample. The reduced flux pinning curves were plotted to study the properties of pinning mechanism of the samples.

\section{RESULTS AND DISCUSSIONS}

\subsection{X-ray diffraction studies}

Since all the samples were prepared from the $\mathrm{MgB}_{2}$ precursor powder, they have shown XRD pattern with all the characteristic peaks corresponding to $\mathrm{MgB}_{2}$ crystal structure as shown in Fig. 1. Although there was a presence of excess Mg during annealing, it is noteworthy to mention that the peak for unreacted Mg was not detected in samples of ArMg and ArHMg. This signifies that Mg diffusing in these samples is used up for filling the Mg deficient sites,

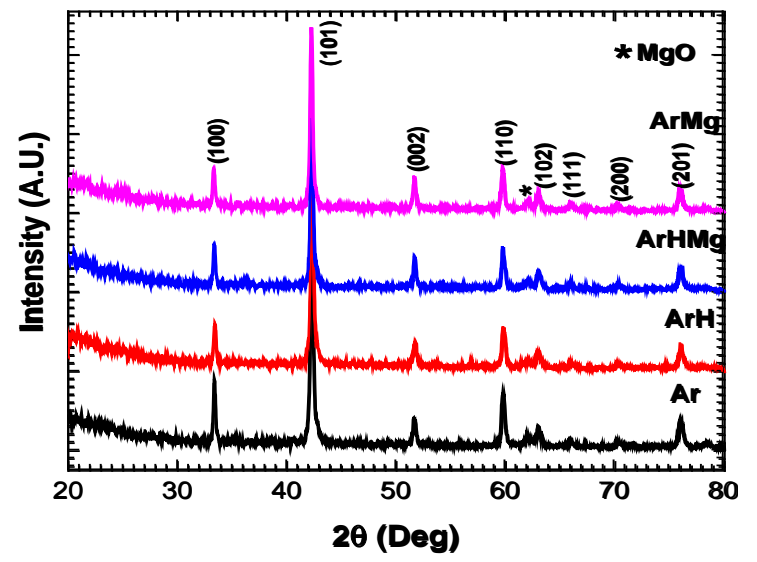

Fig. 1. X-ray diffraction patterns for samples of $\mathrm{Ar}, \mathrm{ArH}$, ArHMg, and ArMg annealed in aforementioned ambiance. rather than settling down on grain boundary or sample surface. There is a small peak for $\mathrm{MgO}$, which cannot be avoided due to high affinity of oxygen towards Mg. There is neither any increase in the MgO peak in the samples annealed in Mg environment. This again shows that there might be a very scarce amount of bare $\mathrm{Mg}$, if not much, settled on the surface otherwise it would have easily got oxidized in the due course giving rise to the MgO concentration.

\subsection{Morphological Studies}

Fig. 2(a) and (b) shows the FE-SEM images of all the samples. From the microstructure, it can be clearly understood that samples annealed in $\mathrm{H}_{2}$ environment have a surface covered with the nm-sized tiny granules. These sites are even more prominent for the samples annealed in combination of $\mathrm{Mg}$ with $\mathrm{Ar}+4 \% \mathrm{H}_{2}$. From XRD, it is clear that there are no peaks observed for unreacted Mg. Also, such kind of sites is observed only in case of samples annealed in $\mathrm{H}_{2}$ environment. Thus it can be argued that $\mathrm{H}_{2}$ being very easily diffusible in the bulk, has some kind of reducing effect on the samples. Since it acts as a strong reducing environment, at high temperatures $\left(900{ }^{\circ} \mathrm{C}\right)$, it may create some fresh boron or $\mathrm{Mg}$ from its oxides or even $\mathrm{MgB}_{2}$ [14] and results in a new nucleation sites at the surfaces. Since MgO is hard to be reduced and elemental $\mathrm{Mg}$ has high vapor pressure, there may be Mg depletion from ArH sample. While, in the case of ArHMg sample, the presence of $\mathrm{Mg}$ in annealing ambiance may support the increase of such kind of sites over the sample surface (As may be seen in Fig. 2(b)). This is in agreement with the
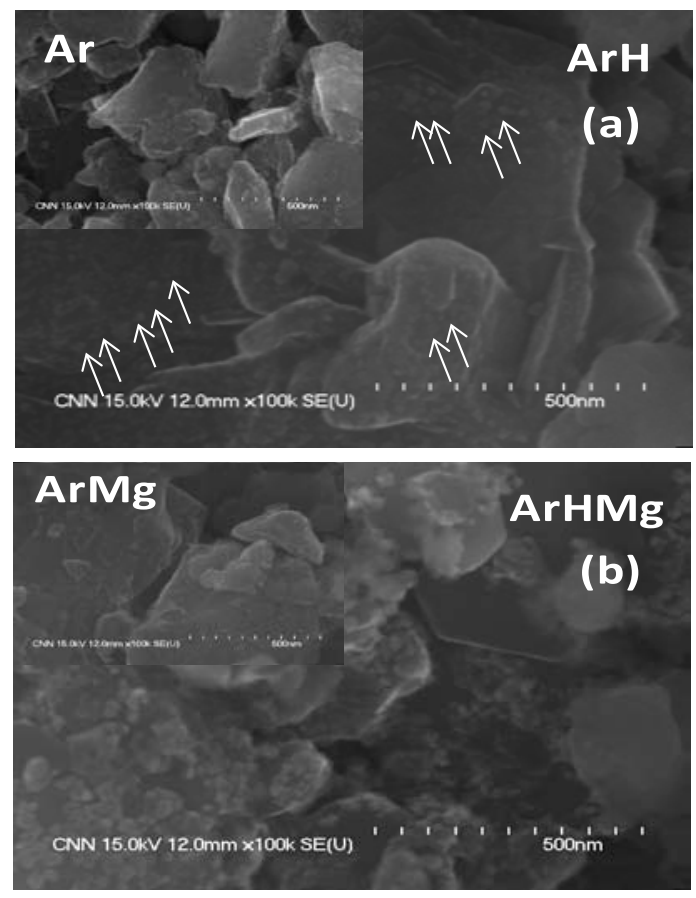

Fig. 2. FE-SEM image of samples (a) annealed in Ar (inset) and $\mathrm{Ar}+4 \% \mathrm{H}_{2}$ (b) annealed in presence of $\mathrm{Mg}$ along with $\mathrm{Ar}$ (inset) and $\mathrm{Ar}+4 \% \mathrm{H}_{2}$. Arrow indicates the presence of nucleation sites observed in the samples annealed in presence of $\mathrm{H}_{2}$ ambiance. (Scale bar is for $500 \mathrm{~nm}$ ). 
results observed for malic acid doped $\mathrm{MgB}_{2}$ wherein the weight fraction of $\mathrm{MgB}_{2}$ was observed to decrease with increase of $\mathrm{H}_{2}$ content in argon [16].

\subsection{Resistivity behavior}

Fig. 3 shows the reduced as well as the original (in inset) resistivity plots for all the samples. From inset it can be easily understood that the overall resistivity decreases from Ar to ArH sample, while it is highly reduced for the samples annealed with $\mathrm{Mg}$ ambiance. The values for effective current carrying area, $A_{\mathrm{f}}$ calculated using Rowell analysis [17-18], onset $\left(T_{\mathrm{c}, \text { on }}\right)$, offset $\left(T_{\mathrm{c} \text {, off }}\right)$ and width $\left(\Delta T_{\mathrm{c}}\right)$ of the superconducting transition is given in table 1 . The decrease in the resistivity of $\mathrm{ArH}$ samples supports our argument of $\mathrm{H}_{2}$ with some reducing effect, which more or less cleans the grain as well as the grain boundary from the oxides rendering the improved overall connectivity and hence the reduced resistivity. This explains the exact scaling of resistivity plots for ArH samples on $\mathrm{Ar}$ in the reduced resistivity plot in Fig. 3.

Meanwhile, in case of samples with Mg ambiance, the resistivity shows much improved power law dependence in temperature with a reduced transition width of $1 \mathrm{~K}$. This can indicate the increased dependence on electron-phonon scattering due to the decrease in defect and improved intra-grain as well as inter-grain connectivity [18]. Further, it can be observed that the actual superconducting current carrying fraction is much higher for ArMg sample as compared to ArHMg sample. This difference is not clearly understood now but can be attributed to the slightly increased grain boundary density of ArHMg due to the formation of new grains at the nucleation sites created by $\mathrm{H}_{2}$ gas as seen in SEM image (Fig. 2(b)).

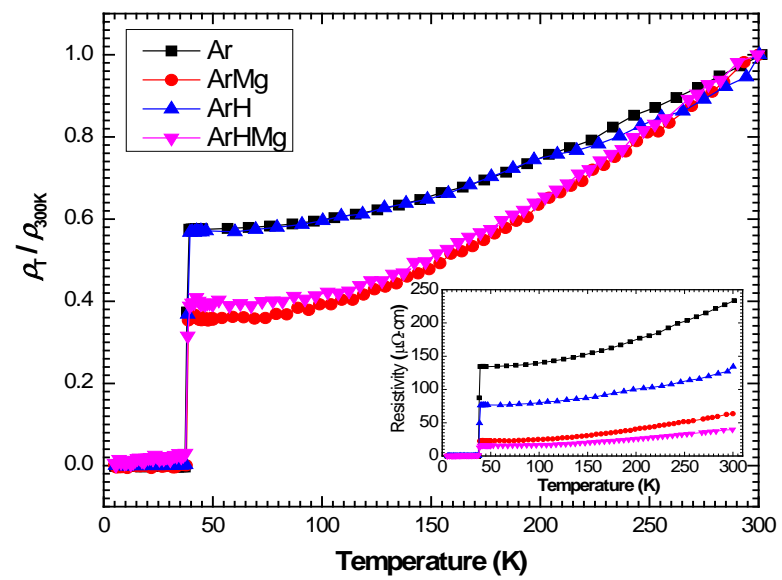

Fig. 3. Reduced and original (inset) temperature dependent resistivity behavior for all the $\mathrm{MgB}_{2}$ samples.

TABLE I

MEASURED VALUES FOR SUPERCONDUCTING AREA FRACTION AND SUPERCONDUCTING TRANSITION PARAMETERS.

\begin{tabular}{ccccc}
\hline Sample & $\mathrm{A}_{\mathrm{f}}$ & $T_{\mathrm{c}, \text { on }}$ & $T_{\mathrm{c}, \text { off }}$ & $\Delta T$ \\
\hline $\mathrm{Ar}$ & 0.07 & 39.2 & 37.2 & 1.98 \\
$\mathrm{ArH}$ & 0.13 & 39.5 & 37.5 & 2 \\
$\mathrm{ArHMg}$ & 0.18 & 38.8 & 37.8 & 1 \\
$\mathrm{ArMg}$ & 0.30 & 38.5 & 37.5 & 1 \\
\hline
\end{tabular}

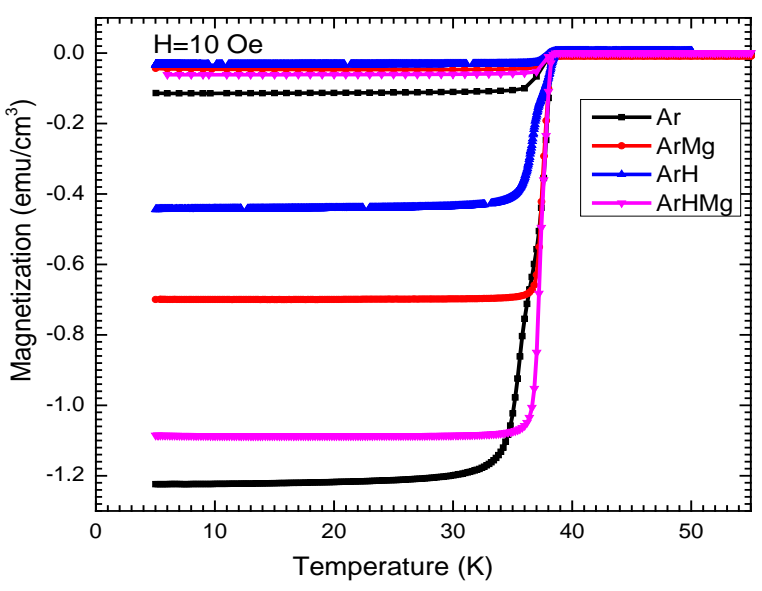

Fig. 4. FC-ZFC plots for all the $\mathrm{MgB}_{2}$ samples.

3.4. Zero Field Cooled (ZFC) and Field Cooled (FC) study

The FC-ZFC plots for all the samples are in a good agreement with the resistivity plots in terms of the superconducting transition temperature. However it is interesting to note that the sample $\mathrm{ArH}$, which shows a lower resistivity values relative to sample Ar, shows very low extent of diamagnetism in Fig. 4. This may indicate that the sample has the lowest density amongst all the samples. This is in support with our argument that $\mathrm{H}_{2}$ has some kind of reducing effect and deplete the level of Mg, which results in the decreased density of the samples. The increase in the extent of diamagnetism in sample ArHMg further consolidates this fact.

\subsection{Magnetization and Critical Current Density}

The magnetization with respect to field (M-H) measurements at $5 \mathrm{~K}$ is shown in Fig. 5 . The plots can be conveniently divided in samples prepared with and without $\mathrm{Mg}$ ambiance. It can be seen that the samples annealed in $\mathrm{Mg}$ ambiance shows wider magnetization loop than those annealed without Mg. Although both the samples show similar M-H response, sample ArHMg seems to be slightly superior than ArMg. Thus even though sample ArH shows very poor performance, the combined effect of $\mathrm{Mg}$ and $\mathrm{H}_{2}$ highly improve the superconducting properties. This effect is reflected straight away in the critical current density curves calculated using equation 1 as shown in Fig. 6.

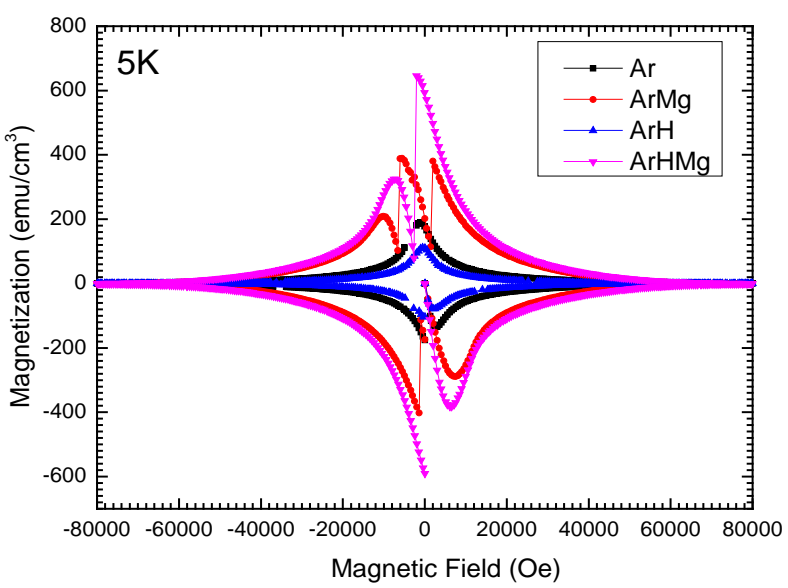

Fig. 5. Magnetization Vs field behavior for all the samples at $5 \mathrm{~K}$. 


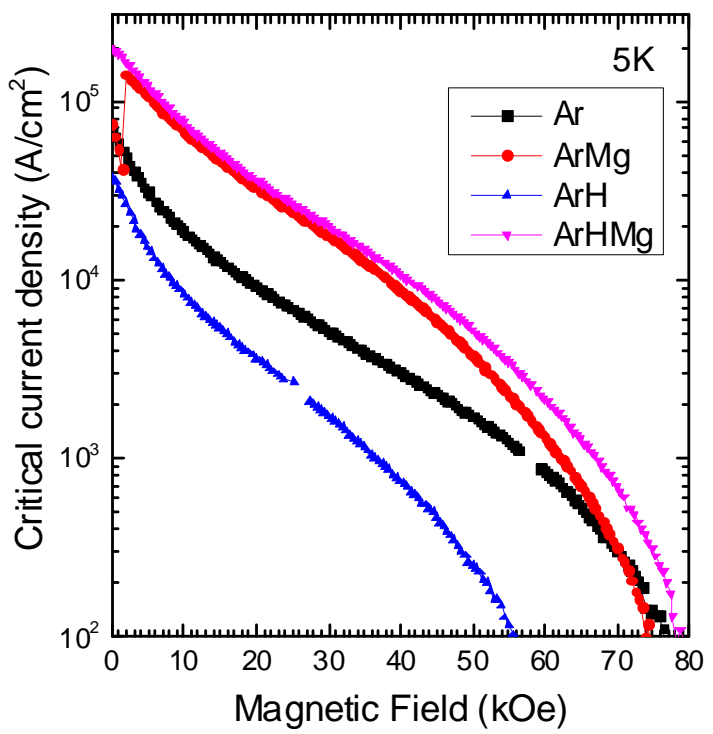

Fig. 6. Critical current density plots at 5K.
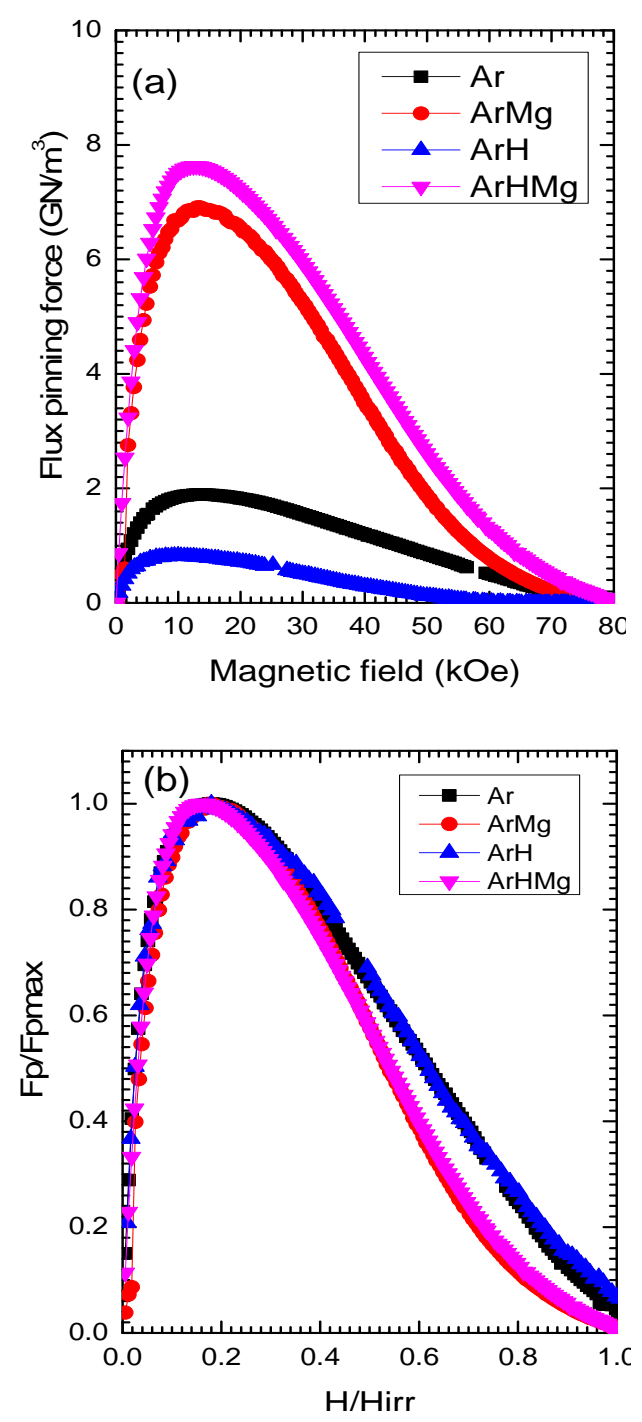

Fig. 7. (a) Flux pinning force plots and (b) Reduced flux pinning force plots for all the samples.
These plots clearly show that the samples treated in $\mathrm{Mg}$ ambiance show the higher performance in overall critical current density values. Here, it is noticeable that the low-field values for ArMg and ArHMg has improved and approach up to $2 \times 10^{5} \mathrm{~A} / \mathrm{cm}^{2}$ as compared to $\mathrm{Ar}$ and $\mathrm{ArH}$ which is less than $10^{5} \mathrm{~A} / \mathrm{cm}^{2}$. The irreversibility field value with the $100 \mathrm{~A} / \mathrm{cm}^{2}$ criteria comes out to be about $80 \mathrm{kOe}$ for all the samples except for ArH which is around $55 \mathrm{kOe}$. Thus it can be understood that although $\mathrm{H}_{2}$ environment alone create a deteriorating effect, but when clubbed with the presence of $\mathrm{Mg}$ the superconducting property of the sample can be enhanced significantly. To understand the pinning mechanism in all these samples, the flux pinning curves were plotted and are as shown in Fig. 7(a) and (b). Fig. 7(a) shows the flux pinning force plots against applied magnetic field. It depicts a clear increase in the flux pinning force in ArMg and ArHMg as compared to Ar and ArH samples. The reduced flux pinning plot for all the samples is shown in Fig. 7(b). It shows maxima around 0.2 for all the samples which implies a prominent grain boundary pinning mechanism which is common for the pure $\mathrm{MgB}_{2}$ bulk samples [19]. The sample treated in the presence of $\mathrm{Mg}$ environment shows an improved flux pinning force. This may be on account of the increased grain boundary density, possibly due to the new grain arrangements caused by nucleation and growth observed especially in combination of $\mathrm{H}_{2}$ and $\mathrm{Mg}$ in annealing ambiance.

\section{CONCLUSION}

It can be concluded that the $\mathrm{Ar}+4 \% \mathrm{H}_{2}$ gas alone act as a reducing environment, which diffuse in the bulk and etch it out. This kind of effect can cause the cleaning of the insulating oxide phases in the sample, most probably at the grain boundary, but at the same time deplete the $\mathrm{Mg}$ from the bulk. So, it happens to decrease the overall resistivity of the sample and adversely affect the critical current density property of $\mathrm{MgB}_{2}$ samples. However, the inclusion of $\mathrm{Mg}$ in this reducing environment may replenish the $\mathrm{Mg}$ deficiencies in the reduced samples and hence improve the overall superconducting properties of $\mathrm{MgB}_{2}$.

\section{ACKNOWLEDGMENT}

This work was supported by KIMS internal program, "Development of Advanced Powder Materials technology for New Growth Engine and Its Transfer to Industry” and by the GRL program (2012-00184).

\section{REFERENCES}

[1] J. Nagamatsu, N. Nakagawa, T. Muranaka, Y. Zenitani, and J. Akimitsu, "Superconductivity at $39 \mathrm{~K}$ in magnesium diboride," Nature, vol. 410, pp. 63-64, 2001.

[2] K.H.P. Kim, J.H. Choi, C.U. Jung, P. Chowdhury, H.S. Lee, M.S. Park, H.J. Kim, J.Y. Kim, Z. Du, E.M. Choi, M.S. Kim, W.N. Kang, S.I. Lee, G.Y. Sung, J.Y. Lee, "Superconducting properties of 
well-shaped $\mathrm{MgB}_{2}$ single crystals,” Phys. Rev. B, vol. 65, pp. 100510, 2002.

[3] A.K. Pradhan, X.Z. Shi, M. Tokunaga, T. Tamegai, Y. Takano, K. Togano, H. Kito, H. Ihara, "Electrical transport and anisotropic superconducting properties in single crystalline and dense polycrystalline $\mathrm{MgB}_{2}$," Phys. Rev. B, vol. 64, pp. 212509, 2001.

[4] A.K. Pradhan, X.Z. Shi, M. Tokunaga, T. Tamegai, Y. Takano, K. Togano, H. Kito, H. Ihara, "Angle-resolved magnetotransport studies in anisotropic $\mathrm{MgB}_{2}$ single crystals," Phys. Rev. B, vol. 65, pp. 144513, 2002

[5] S. Okuma, S. Togo, and K. Amemori, "Observation of superconductivity in thick amorphous $\mathrm{Mg}_{\mathrm{x}} \mathrm{B}_{1-\mathrm{x}}$ films," Phys. Rev. B, vol. 67, pp. 172508, 2003.

[6] B.A. Glowacki, M. Majoros, M. Vickers, J.E. Evetts, Y. Shi and I. McDougall, "Superconductivity of powder-in-tube $\mathrm{MgB}_{2}$ wires," Supercond. Sci. Technol., vol. 14, pp.193-199, 2001.

[7] J. M. Rowell, "The widely variable resistivity of $\mathrm{MgB}_{2}$ samples," Supercond. Sci. Technol., vol. 16, pp. R17-R27, 2003.

[8] P. A. Sharma, N. Hur, Y. Horibe, C. H. Chen, B. G. Kim, S. Guha, Marta Z. Cieplak, and S-W. Cheong, "Percolative superconductivity in $\mathrm{Mg}_{1-\mathrm{x}} \mathrm{B}_{2}$," Phys. Rev. Lett., vol. 89, pp. 167003 , 2002.

[9] P. C. Canfield, D. K. Finnemore, S. L. Bud'ko, J. E. Ostenson, G. Lapertot, C. E. Cunningham, and C. Petrovic. "Superconductivity in dense $\mathrm{MgB}_{2}$ wires,” Phys. Rev. Lett., vol. 86, pp. 2423-2426, 2001.

[10] Y. Bugoslavsky, L. F. Cohen, G. K. Perkins, M. Polichetti, T. J. Tate, R. G. William, and A. D. Caplin, "Enhancement of the high magnetic field critical current density of superconducting $\mathrm{MgB}_{2}$ by proton irradiation," Nature, vol. 411, pp. 561-563, 2001.

[11] D.C. Larbalestier, L. D. Cooley, M. O. Rikel, A. A. Polyanskii, J. Jiang, S. Patnaik, X. Y. Cai, D. M. Feldmann, A. Gurevich, and A. A. Squitieri, "Strongly linked current flow in polycrystalline forms of the superconductor $\mathrm{MgB}_{2}$,” Nature, vol. 410, pp. 186-189, 2001.
[12] T. C. Shields, K. Kawano, D. Holdom, and J. S. Abell, "Microstructure and superconducting properties of hot isostatically pressed $\mathrm{MgB}_{2}$," Supercond. Sci. Technol., vol. 15, pp. 202-205, 2002.

[13] A. Serquis, X. Z. Liao, Y. T. Zhu, J. Y. Coulter, J. Y. Huang, J. O. Willis, D. E. Peterson, F. M. Mueller, N. O. Moreno, J. D. Thompson, V. F. Nesterenko, and S. S. Indrakanti, "Influence of microstructures and crystalline defects on the superconductivity of $\mathrm{MgB}_{2}$," J. Appl. Phys., vol. 92, pp. 351-356, 2002.

[14] Z. K. Liu, D. G. Schlom, Q. Li and X. X. Xi, "Thermodynamics of the Mg-B system: Implications for the deposition of $\mathrm{MgB}_{2}$ thin films,” J. Appl. Phys., vol. 78, pp. 3678-3680, 2001.

[15] C.P. Bean, "Magnetization of hard superconductors," Phys. Rev. Lett., vol. 8, pp. 250-253, 1962.

[16] M. Maeda, J. H. Kim, H. Kumakura, Y. U. Heo, Y. Zhao, Y Nakayama, M. Rindfleisch and S. X. Dou, "Influence of hydrogen containing argon gas on the structural parameters and superconducting properties of malic acid doped $\mathrm{MgB}_{2}$ wires," Scripta Materialia, vol. 64, pp. 1059-1062, 2011.

[17] J. M. Rowell, "The widely variable resistivity of $\mathrm{MgB}_{2}$ sample," Supercond. Sci. Technol., vol. 16, pp. R17-R27, 2003.

[18] S. K. Chen, A. Serquis, G. Serrano, K. A. Yates, M. G. Blamire, D. Guthrie, J. Cooper,H. Wang, S. Margadonna and J. L. MacManus-Driscoll, "Structural and superconducting property variations with nominal $\mathrm{Mg}$ non-stoichiometry in $\mathrm{MgxB}_{2}$ and its enhancement of upper critical field”, Adv. Funct. Mater., vol. 18, pp. 113-120, 2008

[19] F. C. Tarantini, H. U. Aebersold, C. Bernini, V. Braccini, C. Ferdeghini, U. Gambardella, E. Lehmann, P. Manfrinetti, Al Palenzona, I. Pallecchi, M. Vignolo, M. Putti, "Neutron irradiation on $\mathrm{MgB}_{2}$," Physica C, vol. 463-465, pp. 211-215, 2007. 